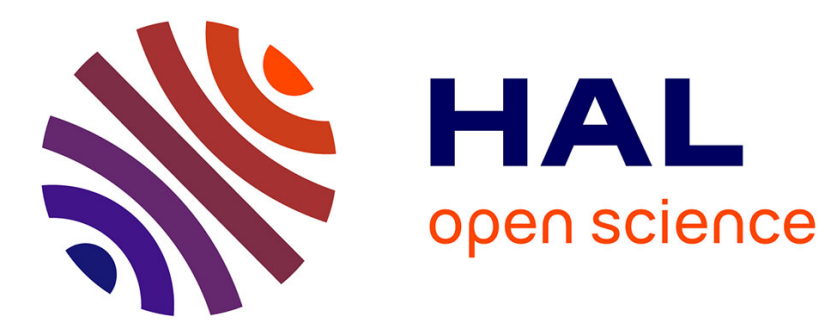

\title{
Power fluctuations in turbulence
}

Wouter J. T. Bos, Rémi Zamansky

\section{To cite this version:}

Wouter J. T. Bos, Rémi Zamansky. Power fluctuations in turbulence. Physical Review Letters, 2019, 122 (12), pp.1-6. 10.1103/physrevlett.122.124504 . hal-02134629

\section{HAL Id: hal-02134629 \\ https://hal.science/hal-02134629}

Submitted on 20 May 2019

HAL is a multi-disciplinary open access archive for the deposit and dissemination of scientific research documents, whether they are published or not. The documents may come from teaching and research institutions in France or abroad, or from public or private research centers.
L'archive ouverte pluridisciplinaire HAL, est destinée au dépôt et à la diffusion de documents scientifiques de niveau recherche, publiés ou non, émanant des établissements d'enseignement et de recherche français ou étrangers, des laboratoires publics ou privés. 


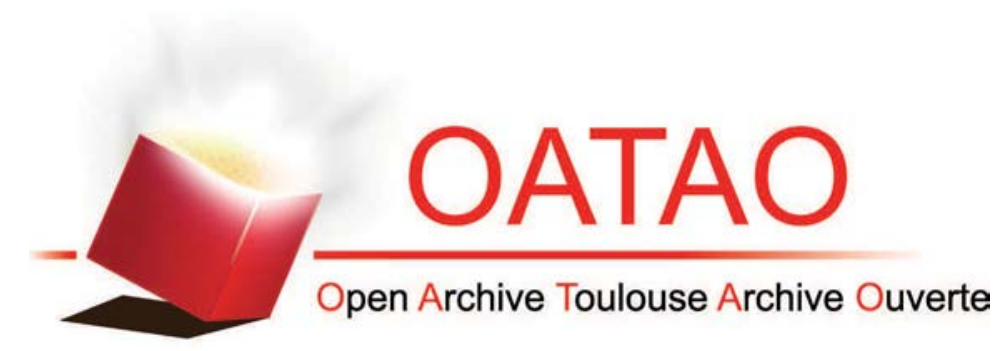

\section{Open Archive Toulouse Archive Ouverte (OATAO)}

OATAO is an open access repository that collects the work of some Toulouse researchers and makes it freely available over the web where possible.

This is an author's version published in: https://oatao.univ-toulouse.fr/23853

Official URL : https://doi.org/10.1103/physrevlett.122.124504

\section{To cite this version :}

Bos, Wouter J. T. and Zamansky, Rémi Review Letters, 122 (12). 1-6. ISSN 0031-9007

Any correspondence concerning this service should be sent to the repository administrator: tech-oatao@listes-diff.inp-toulouse.fr 


\title{
Power fluctuations in turbulence
}

\author{
Wouter J. T. Bos ${ }^{1}$ and Rémi Zamansky ${ }^{2}$ \\ ${ }^{1}$ LMFA, CNRS, Ecole Centrale de Lyon, Université de Lyon, Ecully 69134, France \\ ${ }^{2}$ Institut de Mécanique des Fluides de Toulouse (IMFT), Université de Toulouse, CNRS, Toulouse 31400, France
}

\begin{abstract}
To generate or maintain a turbulent flow, one needs to introduce kinetic energy. This energy injection necessarily fluctuates and these power fluctuations act on all turbulent excited length scales. If the power is injected using forces proportional to the velocity, such as those common in shear flows, or with a force acting at the largest scales only, the spectrum of these fluctuations is shown to have a universal inertial range, proportional to the energy spectrum.
\end{abstract}

DOI: 10.1103/PhysRevLett.122.124504

During the famous 1961 conference on turbulence, convincing evidence was presented in support of Kolmogorov's 1941 theory [1] of turbulence. Indeed a measurement of the energy spectrum $E(k)$ in a tidal channel [2] showed a power law scaling proportional to $k^{-5 / 3}$. At the same conference, Kolmogorov himself presented a correction to his theory [3]. Inspired by a remark of Landau, he illustrated that the 1941 picture of an inertial range depending on the mean energy flux was flawed, since large spatiotemporal fluctuations of the energy flux, determined by the energy injection mechanism, should lead to a correction of the $-5 / 3$ power law scaling. It was realized that scaling relations could depend on the, necessarily nonuniversal, energy injection mechanism.

However, the energy injection might be more universal than perhaps expected. The reason for this is that the energy input is not only determined by the precise input mechanism, but also by the impedance of the flow itself. Clearly, a force cannot work if it is applied orthogonal to the velocity of a fluid element. The work done by a force through its interaction with a flow is investigated in the present Letter.

The fluctuations of the energy input, or power, have been studied previously, in particular in experimental flows between rotating disks [4,5]. Some universality was observed in the shape of the probability density functions (PDFs) [6,7]. A model for the shape of these PDFs could be derived using a Gaussian assumption [8]. Further investigations of the power fluctuations include a numerical study of shear flow [9] and an investigation of the particular case of wave turbulence [10]. More recently the study of power fluctuations was revisited in the context of Lagrangian statistics [11,12]. The related quantity of subgrid-flux fluctuations, important for large eddy simulation strategies, was considered in some detail $[13,14]$.

Most of these studies investigated the PDF of the power fluctuations, not their spectrum. Only minor attention was given to temporal spectra in experiments [15]. In wind farms, the spectral distribution of the power spectrum has received some more attention, since electricity fluctuations are directly related to the power fluctuations in the incoming turbulence [16-20]. These studies exclusively focus on the time domain.

We will focus here on the wave number spectrum of the power fluctuations in turbulence using theoretical considerations and direct numerical simulations (DNSs). It will be shown that the power fluctuation spectrum contains a universal scaling range at high Reynolds numbers even when the probability density function of the fluctuations can differ from flow to flow.

We consider the incompressible Navier-Stokes equations, and in particular the fluctuations of the velocity $u_{i}=U_{i}-\left\langle U_{i}\right\rangle$, where $U_{i}(\boldsymbol{x}, t)$ is the velocity field and the angular brackets denote an ensemble average. In a statistically homogeneous flow the fluctuations are governed by

$$
\partial_{t} u_{i}+\partial_{j}\left(u_{i} u_{j}\right)=-\partial_{i} p+\nu \Delta u_{i}+f_{i}
$$

combined with the constraint $\partial_{i} u_{i}=0$. The fluctuating pressure is indicated by $p, \nu$ is the kinematic viscosity and $f$ is the applied force field per unit mass of fluid. The kinetic energy balance takes a particularly simple form

$$
d_{t}\langle K\rangle=\langle P\rangle-\langle\epsilon\rangle
$$

where the energy is given by $K=u_{i} u_{i} / 2$ and $\epsilon$ is the dissipation and the energy injection is given by,

$$
P=u_{i} f_{i}
$$

Obviously in a steady state we have $\langle P\rangle=\langle\epsilon\rangle$, But locally there is no reason for $P=\epsilon$. We can define the fluctuations by $P^{\prime}=P-\langle P\rangle$ and $\epsilon^{\prime}=\epsilon-\langle\epsilon\rangle$, and it is in particular $P^{\prime}$ that we will focus on.

Let us start by answering the following question: is it possible to define a forcing such that $P^{\prime}$ is zero everywhere? 
In other words, is it possible to inject the same amount of energy uniformly in space? Since it is only possible to inject energy if the scalar product of the velocity and the applied force is nonzero [see Eq. (3)], it is impossible to inject energy in stagnation points of the flow. Furthermore, since $u_{i}$ is a continuous, zero-mean quantity, stagnation points will exist and, at these points, no energy can be injected. Therefore, even if we control perfectly the applied force, in practice no turbulent flow can exist without power fluctuations. Power fluctuations are thus ubiquitous and we will investigate some of their properties in the following.

We will in the following consider the forcing

$$
f_{i}=\alpha_{i j} u_{j}+\beta_{i}
$$

where $\alpha_{i j}$ and $\beta_{i}$ are both functions of space and time, and where $\boldsymbol{\beta}$ is the component of the force orthogonal to the local velocity $\boldsymbol{u}$. It is thus the part that does not inject energy. This forcing is fairly general. For instance, in a large class of turbulent flows the energy of the fluctuating turbulent field is supplied by the average velocity field, which corresponds to the case $\alpha_{i j}=\partial_{j}\left\langle U_{i}\right\rangle$. The linear forcing scheme, widely used in DNS [21] corresponds to $\alpha_{i j}=\alpha \delta_{i j}$.

We will consider the specific case where $\alpha$ is not determined by the fluctuating flow properties, but only by externally imposed parameters. The case of homogeneous shear flow is an academic example of such a forcing. The average injected energy is then given by

$$
\langle P\rangle=\alpha_{i j}\left\langle u_{i} u_{j}\right\rangle
$$

and evidently $\boldsymbol{\beta}$ does not appear in this expression. The variance of the fluctuations is given by

$$
\left\langle P^{\prime 2}\right\rangle=\alpha_{i j} \alpha_{m n}\left\langle u_{i} u_{j} u_{m} u_{n}\right\rangle-\langle P\rangle^{2}
$$

Working out a Gaussian estimate for the fourth order velocity correlations, we find

$I_{P} \equiv \frac{\left\langle P^{\prime 2}\right\rangle}{\langle P\rangle^{2}}=\frac{\alpha_{i j} \alpha_{m n}\left(\left\langle u_{i} u_{m}\right\rangle\left\langle u_{j} u_{n}\right\rangle+\left\langle u_{i} u_{n}\right\rangle\left\langle u_{j} u_{m}\right\rangle\right)}{\langle P\rangle^{2}}$.

For the linear forcing we find then, using isotropy, $I_{P}=2 / 3$, whereas for homogeneous shear $\partial_{z}\left\langle U_{x}\right\rangle$ we have

$$
I_{P}=1+\rho_{u w}^{-2}
$$

with $\rho_{u w}=\langle u w\rangle /\left(\left\langle u^{2}\right\rangle^{1 / 2}\left\langle w^{2}\right\rangle^{1 / 2}\right)$, where $u$ and $w$ are velocity fluctuations in the streamwise and cross-stream direction, respectively. We have therefore the interesting feature that the energy injection intensity is directly related to the anisotropy of the flow. In experiments and simulations of homogeneous shear flow [22] $\rho_{u w}=\mathcal{O}(0.5)$ so that $I_{P}$ is around 5. The Gaussian estimate shows therefore that the injection intensity can strongly vary between different flow types. What we will now investigate is how these fluctuations vary as a function of scale.

The fluctuation spectrum is defined as

$$
E_{P}(k)=\int_{|\boldsymbol{k}|=k}\left\langle P^{\prime}(\boldsymbol{k}) P^{\prime}(-\boldsymbol{k})\right\rangle d S_{k}
$$

where the integration is performed over spherical shells of radius $k$ (the wave number) such that

$$
\int E_{P}(k) d k=\left\langle P^{\prime 2}\right\rangle
$$

In these and following expressions, Fourier coefficient are recognized by their argument $(\boldsymbol{k}, \boldsymbol{p}$, or $\boldsymbol{q})$. The power spectrum writes then

$$
\begin{aligned}
E_{P}(k)= & \int_{|\boldsymbol{k}|=k} \iint\left\langle f_{i}(\boldsymbol{p}) u_{i}(\boldsymbol{k}-\boldsymbol{p}) f_{j}(\boldsymbol{q}) u_{j}(-\boldsymbol{k}-\boldsymbol{q})\right\rangle \\
& \times d \boldsymbol{p} d \boldsymbol{q} d S_{k} .
\end{aligned}
$$

Without further information on the nature of the forcing function $\boldsymbol{f}$, we cannot make any predictions on the scaling of the spectrum $E_{P}(k)$. We will therefore consider the following two specific cases. The first one is a compact forcing at the large scales. The second one is a forcing reminiscent of the influence of a mean velocity gradient on a turbulent flow.

In the first case, where the forcing is confined to the largest scales, or smallest wave numbers, we can follow a reasoning similar to the one of Batchelor, Howells, and Townsend in deriving the shape of the temperature spectrum in low Prandtl number convection [23]. Let us consider that $\boldsymbol{f}$ acts at the smallest wave numbers around $|\boldsymbol{k}|=k_{f}$, while we are interested in the scaling of $E_{P}(k)$ for $k \gg k_{f}$. In this range of scales $(|\boldsymbol{k}-\boldsymbol{p}| \gg|\boldsymbol{p}|)$ we can assume statistical independence of the velocity mode $f_{i}(\boldsymbol{p})$ and $u_{i}(\boldsymbol{k}-\boldsymbol{p})$, so that we can split the fourth order correlation,

$$
\begin{aligned}
E_{P}(k) \approx & \int_{|\boldsymbol{k}|=k} \iint\left\langle f_{i}(\boldsymbol{p}) f_{j}(\boldsymbol{q})\right\rangle\left\langle u_{i}(\boldsymbol{k}-\boldsymbol{p}) u_{j}(-\boldsymbol{k}-\boldsymbol{q})\right\rangle \\
& \times d \boldsymbol{p} d \boldsymbol{q} d S_{k} .
\end{aligned}
$$

Furthermore, orthogonality of Fourier coefficients, and $\boldsymbol{k}-\boldsymbol{p} \approx \boldsymbol{k}$ leads to,

$$
E_{P}(k) \approx\left\langle f_{i} f_{j}\right\rangle \phi_{i j}(k)
$$

where $\phi_{i j}(k)=\int_{|\boldsymbol{k}|=k}\left\langle u_{i}(\boldsymbol{k}) u_{j}(-\boldsymbol{k})\right\rangle d S_{k}$ and $\phi_{i i}(k)=2 E(k)$. We see that when the forcing is constrained to the large scales, the power-spectrum is proportional to the spectral tensor $\phi_{i j}(k)$. In the case of an isotropic forcing, this shows that 


$$
E_{P}(k) \approx \frac{2}{3}\left\langle f_{i} f_{i}\right\rangle E(k)
$$

This result shows that, for an isotropic large-scale forcing, the power spectrum scales proportional to the energy spectrum. The fluctuations of the energy injection remain thus not confined to the forced scales but are distributed over all scales.

We can now ask what the relevance of this result is in the case where the forcing is not an artificial forcing confined around $k_{f}$, but where the kinetic energy is generated through the interaction of the turbulence with an externally imposed velocity gradient. In that case we cannot directly assume independence of the forcing spectrum and the velocity modes at large wave numbers in order to simplify Eq. (11). Let us consider the simplest case, $f_{i}=\alpha u_{i}$, corresponding to a linear forcing, acting on all scales. Since we have in this case a direct relation between the power and the kinetic energy, $P=f_{i} u_{i}=2 \alpha K$, the power spectrum is now,

$$
E_{P}(k)=4 \alpha^{2} E_{K}(k),
$$

where $E_{K}(k)$ is the kinetic energy fluctuation spectrum such that,

$$
\int E_{K}(k) d k=\left\langle(K-\langle K\rangle)^{2}\right\rangle
$$

Since the integral of this spectrum yields the square of the kinetic energy fluctuations, a fourth order velocity correlation, one might expect that it does not scale like the kinetic energy spectrum, which is associated with a second order velocity correlation. This is actually not so, and the asymptotic wave number dependence of both spectra is the same. Indeed, the spectrum $E_{K}(k)$ has received some attention in the past [24-27], and is known to scale as

$$
E_{K}(k) \sim\left\langle u_{i} u_{i}\right\rangle E(k) .
$$

Therefore, even in this case where the forcing is not constrained to the largest scales, the small scales are obeying a sweeping scaling [28]. Such results are fairly robust, even when flows are not strictly isotropic. Indeed, the first evidence of the validity of Gaussian (or sweeping) scaling for higher-order spectra was obtained in mixing layer and atmospheric boundary layer flows [24]. We will check these ideas now.

We carried out direct numerical simulations (DNS) of isotropic turbulence in a periodic box at a resolution of $512^{3}$ grid points. Details of the pseudospectral code can be found in Refs. [29,30]. Three different types of forcing were considered. $f_{1}$ : a deterministic forcing keeping the volume-averaged energy at the large scales constant [31]. This forcing was applied at the wave modes with $k<2 \sqrt{2}$. $f_{2} .:$ a stochastic forcing where the energy injection is given by an Ornstein-Uhlenbeck process [32], also applied at the wave modes with $k<2 \sqrt{2}$. $f_{3}$ : a linear forcing, $f_{i}=\alpha u_{i}$ [21,33] applied to all scales. These forcing procedures allow the turbulence to reach a statistically steady state with a Reynolds number $R_{\lambda} \approx 130$ along with $\eta / \Delta x \approx 0.75$, with $\eta=\nu^{3 / 4} / \epsilon^{1 / 4}$.

In our simulations a close to statistically steady state is obtained where the mean production equals approximately the mean dissipation. The normalized variance of the dissipation rate fluctuations $\left\langle\epsilon^{\prime 2}\right\rangle /\langle\epsilon\rangle^{2} \approx 1.6$ for the three flows. The values of the variance of the power fluctuations are however significantly different, $\left\langle P^{\prime 2}\right\rangle /\langle\epsilon\rangle^{2}=$ $1.1 ; 4.2 ; 0.8$ (for $f_{1} ; f_{2} ; f_{3}$, respectively). This difference is also illustrated in the visualizations of the power during the steady state, shown in Fig. 1. It is observed that the power fields are qualitatively different. For instance, in the third forcing scheme the injected power can only be positive, whereas negative values are observed in the other two cases.

This is further illustrated in Fig. 2(a), where it is observed that the PDFs of the power are very different in shape. On the contrary, the PDFs of the dissipation rate fluctuations are nearly identical (not shown). Analytical expressions can be derived for the power fluctuation PDFs assuming Gaussianity of the velocity fluctuations, at least for forcing $f_{3}$, where the shape of the PDF should resemble a $\chi^{2}$ distribution. We will not further focus on this, but we stress that the shapes of the PDFs indicate that the power fluctuations generated by the three different forcing schemes are quite different.

In Fig. 2(b) it is shown that the length scale distribution is not so different. The spectra of forcing scheme 1 and 2 are very similar, with a power law exponent close to $-5 / 3$. The third forcing yields a power fluctuation spectrum that is shallower, with a slope close to -1 . It seems thus that for the first two forcing schemes, which are confined to the largest scales, the predicted scaling is observed. However, for the linear forcing, applied at all scales, a strong deviation is observed. In the following we will try to understand this difference. In particular we will show that also for this case a $-5 / 3$ scaling is expected to appear, be it at higher Reynolds numbers.

The statistical independence, invoked to derive the scaling relations is in the present case equivalent to the
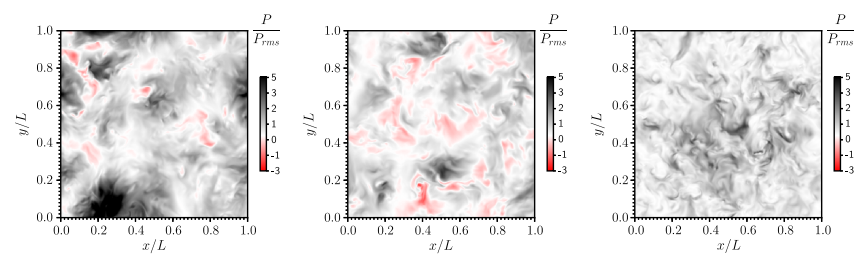

FIG. 1. Visualizations of the power input in the turbulent flows. From left to right forcing schemes $f_{1}, f_{2}$, and $f_{3}$. 

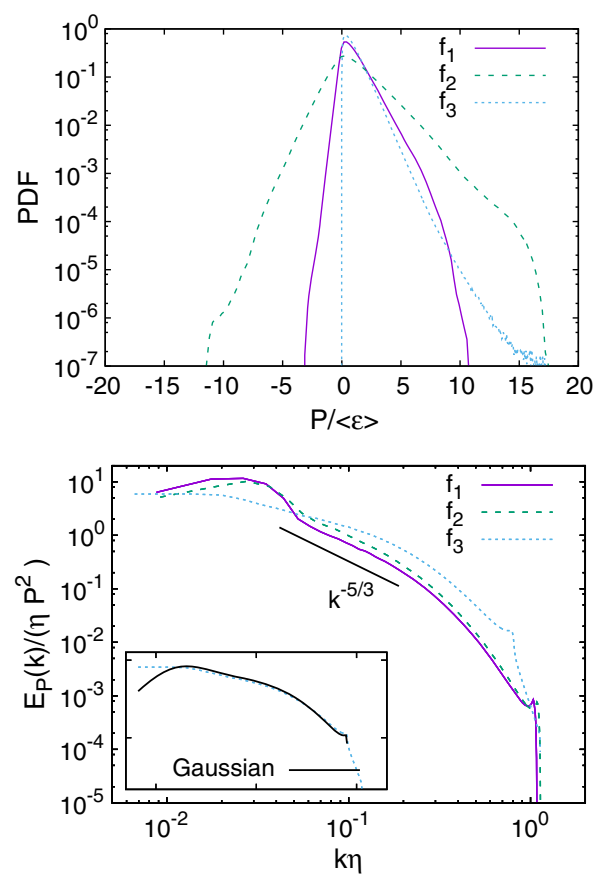

FIG. 2. (a) DNS results for the PDFs of the power fluctuations for three different forcing schemes. (b) Power fluctuation spectra for the same three cases. In the inset we compare the spectrum for forcing $f_{3}$ with its Gaussian estimate.

assumption of Gaussianity. Indeed, the sweeping scaling was derived by assuming joint-Gaussian velocity statistics $[24,27]$. Even though Gaussian estimates give for certain quantities good order-of-magnitude estimates, certain features are completely missed. For instance, the net energy transfer between scales is zero for joint-Gaussian velocity fluctuations. Furthermore, the dissipation rate fluctuations are severely mispredicted if Gaussianity is assumed [34-36]. Is this the case here?

The Gaussian estimate of the power spectrum for linearly forced isotropic turbulence is

$$
E_{P}(k)=2 k \alpha^{2} \int_{\Delta}\left(1-x^{2}\right) E(p) E(q) \frac{d p}{p} \frac{d q}{q},
$$

where $x=(\boldsymbol{p} \cdot \boldsymbol{q}) / p q$ and $\Delta$ indicates a subdomain in the $\boldsymbol{p}$, $\boldsymbol{q}$ plane where the wave vectors $\boldsymbol{k}, \boldsymbol{p}, \boldsymbol{q}$ can form a triangle. This integral can be evaluated numerically for a given energy spectrum. We have, to check the assumption of Gaussianity, compared the power spectrum from the DNS with the Gaussian prediction, using in Eq. (18) the energy spectrum computed from the DNS data. The comparison is shown in the inset of Fig. 2(b). The Gaussian prediction, computed from the DNS energy spectrum, very accurately collapses with the actual power spectrum, except for the smallest wave numbers. This different behavior is due to the different nature of the boundary conditions in the two approaches. Indeed, the theoretical, integral Gaussian estimate [Eq. (18)] assumes an infinite domain and

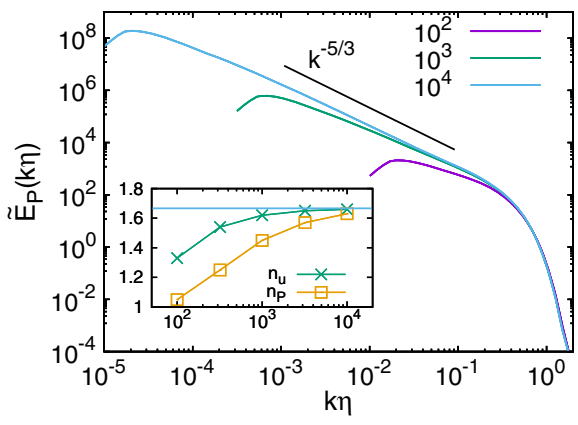

FIG. 3. Gaussian estimates for $E_{p}(k)$, computed from spectral closure results using Eq. (18). Inset: spectral index $n_{u}$ of the inertial range scaling of the energy spectrum and $n_{P}$, corresponding to the power law exponent of the power fluctuation spectrum as a function of the Reynolds number $R_{\lambda}$.

periodicity is not taken into account. Nevertheless, for all wave numbers in the inertial and dissipation range, the agreement is very good.

The actual reason that the power spectrum for the linear forcing is not proportional to $k^{-5 / 3}$ is that universal scaling appears at higher Reynolds numbers. In order to illustrate this, we carried out closure simulations of the eddy damped quasi normal Markovian type. Indeed the convergence to asymptotic statistics, as compared to experiments or simulations, is well predicted by this closure scheme [37]. We have computed, using the linear forcing, steady states at Reynolds numbers ranging from 100 to $10^{4}$. We used the energy spectra from these simulations to compute the Gaussian estimate of the power spectrum. The results are shown in Fig. 3. It is shown that a good collapse is possible if the spectra are normalized by the mixed scaling [Eq. (14)], involving $\left\langle u_{i} u_{i}\right\rangle, \epsilon$, and $\eta$. Indeed, in Fig. 3 we plot

$$
\tilde{E_{P}}=\frac{E_{P}(k \eta)}{\alpha^{2}\left\langle u_{i} u_{i}\right\rangle \epsilon^{2 / 3} \eta^{5 / 3}},
$$

and the good collapse of these normalized spectra shows the validity of Eq. (14). Furthermore it is observed that for the closure results, the convergence of the spectrum to asymptotic scaling is very slow. In the inset of Fig. 3 it is observed that at $R_{\lambda}=100$ the power law exponent is close to -1 , as for the DNS, but that the spectrum becomes gradually steeper, attaining close to asymptotic scaling around $R_{\lambda}=10^{4}$. DNS at higher resolution and Reynolds number should be able to assess this tendency to some extent, since the largest simulations of isotropic turbulence [38] allow currently to reach Reynolds numbers of the order of $R_{\lambda} \approx 2 \times 10^{3}$.

With respect to the general applicability of the present insights one could object that the linear forcing $f_{i}=\alpha u_{i}$ is rather artificial, but the ideas can be transposed to shear flow. Indeed, shear is the principal turbulent kinetic energy injection mechanism in the absence of body forces. In the 
case of steady uniform shear $S \equiv \partial_{z}\left\langle U_{x}\right\rangle$, the energy input is $P=-S\langle u w\rangle$. The power spectrum for this case is $E_{P}(k)=S^{2} E_{u w}(k)$, where the shear-stress fluctuation spectrum defined as

$$
\int E_{u w}(k) d k=\left\langle(u w)^{2}\right\rangle-\langle u w\rangle^{2}
$$

It was shown $[39,40]$ that also this spectrum is proportional to the trace of the spectral tensor and thereby scales as $k^{-5 / 3}$, so that the above arguments for isotropic turbulence can be transposed to the very important case of shear flow.

We showed that for a turbulent flow displaying the Kolmogorov energy spectrum, the power spectrum will reflect this scaling, following Eq. (14). Thereby the input fluctuations have a universal equilibrium range. Of course, it is possible to define a forcing function that will lead to different scaling, for instance, a forcing spectrum with a peak at the large wave numbers. However, as long as the support of the forcing function is confined to large wave numbers, or with a power-law spectrum steeper than $k^{-1}$, the power spectrum will be given by Eq. (14). The next step in understanding turbulence intermittency is now the investigation of the fluctuations of the energy flux and its relation to the power fluctuations in order to understand if universality is also conserved for that quantity. The answer to that question definitely needs further research.

This work was performed using HPC resources from GENCI-CINES.

[1] A. N. Kolmogorov, The local structure of turbulence in incompressible viscous fluid for very large Reynolds numbers, Dokl. Akad. Nauk SSSR 30, 301 (1941).

[2] H. L. Grant, R. W. Stewart, and A. Moilliet, Turbulence spectra from a tidal channel, J. Fluid Mech. 12, 241 (1962).

[3] A. N. Kolmogorov, A refinement of previous hypotheses concerning the local structure of turbulence in a viscous incompressible fluid at high Reynolds number, J. Fluid Mech. 13, 82 (1962).

[4] R. Labbé, J.-F. Pinton, and S. Fauve, Power fluctuations in turbulent swirling flows, J. Phys. II 6, 1099 (1996).

[5] O. Cadot, Y. Couder, A. Daerr, S. Douady, and A. Tsinober, Energy injection in closed turbulent flows: Stirring through boundary layers versus inertial stirring, Phys. Rev. E 56, 427 (1997).

[6] S. T. Bramwell, P. C. W. Holdsworth, and J.-F. Pinton, Universality of rare fluctuations in turbulence and critical phenomena, Nature (London) 396, 552 (1998).

[7] J.-F. Pinton, P. C. W. Holdsworth, and R. Labbé, Power fluctuations in a closed turbulent shear flow, Phys. Rev. E 60, R2452 (1999).

[8] M. M. Bandi, S. G. Chumakov, and C. Connaughton, Probability distribution of power fluctuations in turbulence, Phys. Rev. E 79, 016309 (2009).
[9] J. Schumacher and B. Eckhardt, Fluctuations of energy injection rate in a shear flow, Physica (Amsterdam) 187D, 370 (2004).

[10] E. Falcon, S. Aumaître, C. Falcón, C. Laroche, and S. Fauve, Fluctuations of Energy Flux in Wave Turbulence, Phys. Rev. Lett. 100, 064503 (2008).

[11] H. Xu, A. Pumir, G. Falkovich, E. Bodenschatz, M. Shats, H. Xia, N. Francois, and G. Boffetta, Flight-crash events in turbulence, Proc. Natl. Acad. Sci. U.S.A. 111, 7558 (2014).

[12] A. Pumir, H. Xu, G. Boffetta, G. Falkovich, and E. Bodenschatz, Redistribution of Kinetic Energy in Turbulent Flows, Phys. Rev. X 4, 041006 (2014).

[13] S. Cerutti and C. Meneveau, Intermittency and relative scaling of subgrid-scale energy dissipation in isotropic turbulence, Phys. Fluids 10, 928 (1998).

[14] T. Aoyama, T. Ishihara, Y. Kaneda, M. Yokokawa, K. Itakura, and A. Uno, Statistics of energy transfer in highresolution direct numerical simulation of turbulence in a periodic box, J. Phys. Soc. Jpn. 74, 3202 (2005).

[15] J. H. Titon and O. Cadot, The statistics of power injected in a closed turbulent flow: Constant torque forcing versus constant velocity forcing, Phys. Fluids 15, 625 (2003).

[16] P. Milan, M. Wächter, and J. Peinke, Turbulent Character of Wind Energy, Phys. Rev. Lett. 110, 138701 (2013).

[17] R. Calif and F. G. Schmitt, Multiscaling and joint multiscaling description of the atmospheric wind speed and the aggregate power output from a wind farm, Nonlinear Processes Geophys. 21, 379 (2014).

[18] K. Viestenz and R. B. Cal, Streamwise evolution of statistical events in a model wind-turbine array, BoundaryLayer Meteorol. 158, 209 (2016).

[19] M. M. Bandi, Spectrum of Wind Power Fluctuations, Phys. Rev. Lett. 118, 028301 (2017).

[20] J. Bossuyt, C. Meneveau, and J. Meyers, Wind farm power fluctuations and spatial sampling of turbulent boundary layers, J. Fluid Mech. 823, 329 (2017).

[21] T. Lundgren, Linearly forced isotropic turbulence, Annual Research Briefs 461 (2003).

[22] S. B. Pope, Turbulent Flows (Cambridge University Press, Cambridge, 2000).

[23] G. K. Batchelor, I. D. Howells, and A. A. Townsend, Smallscale variation of convected quantities like temperature in turbulent fluid. Part 2, J. Fluid Mech. 5, 134 (1959).

[24] C. W. Van Atta and J. C. Wyngaard, On higher-order spectra of turbulence, J. Fluid Mech. 72, 673 (1975).

[25] M. Nelkin and M. Tabor, Time correlations and random sweeping in isotropic turbulence, Phys. Fluids A 2, 81 (1990).

[26] T. Ishihara, Y. Kaneda, M. Yokokawa, K. Itakura, and A. Uno, Spectra of energy dissipation, enstrophy and pressure by high-resolution direct numerical simulations of turbulence in a periodic box, J. Phys. Soc. Jpn. 72, 983 (2003).

[27] W. J. T. Bos and R. Rubinstein, On the strength of the nonlinearity in isotropic turbulence, J. Fluid Mech. 733, 158 (2013).

[28] H. Tennekes, Eulerian and Lagrangian time microscales in isotropic turbulence, J. Fluid Mech. 67, 561 (1975). 
[29] R. Zamansky, F. Coletti, M. Massot, and A. Mani, Turbulent thermal convection driven by heated inertial particles, J. Fluid Mech. 809, 390 (2016).

[30] M. Gorokhovski and R. Zamansky, Modeling the effects of small turbulent scales on the drag force for particles below and above the Kolmogorov scale, Phys. Rev. Fluids 3, 034602 (2018).

[31] S. Chen, G. D. Doolen, R. H. Kraichnan, and Z.-S. She, On statistical correlations between velocity increments and locally averaged dissipation in homogeneous turbulence, Phys. Fluids A 5, 458 (1993).

[32] V. Eswaran and S. B. Pope, An examination of forcing in direct numerical simulations of turbulence, Comput. Fluids 16, 257 (1988).

[33] C. Rosales and C. Meneveau, Linear forcing in numerical simulations of isotropic turbulence: Physical space implementations and convergence properties, Phys. Fluids 17, 095106 (2005).

[34] H. Chen, J. R. Herring, R. M. Kerr, and R. H. Kraichnan, Non-Gaussian statistics in isotropic turbulence, Phys. Fluids A 1, 1844 (1989).
[35] T. Gotoh and R. S. Rogallo, Intermittency and scaling of pressure at small scales in forced isotropic turbulence, $\mathrm{J}$. Fluid Mech. 396, 257 (1999).

[36] W. J. T. Bos, R. Chahine, and A. V. Pushkarev, On the scaling of temperature fluctuations induced by frictional heating, Phys. Fluids 27, 095105 (2015).

[37] W. J. T. Bos, H. Touil, and J.-P. Bertoglio, Reynolds number dependency of the scalar flux spectrum in isotropic turbulence with a uniform scalar gradient, Phys. Fluids 17, 125108 (2005).

[38] T. Ishihara, K. Morishita, M. Yokokawa, A. Uno, and Y. Kaneda, Energy spectrum in high-resolution direct numerical simulations of turbulence, Phys. Rev. Fluids 1, 082403 (2016).

[39] W. K. George, P. D. Beuther, and R. E. A. Arndt, Pressure spectra in turbulent free shear flows, J. Fluid Mech. 148, 155 (1984).

[40] C. Tong, Spectra of scalar-flux and stress fluctuations in the atmospheric surface layer, J. atmos. Sci. 54, 1277 (1997). 